\title{
Changes and Clinical Significance of PIVKA-II in Hepatitis E Patients
}

\author{
Youran Chen ${ }^{1 \dagger}$, Yanyan Yang ${ }^{1+}$, Shanshan $\mathrm{Li}^{1}$, Minghao Lin ${ }^{1}$, Xueting Xie ${ }^{1}$, Huifang Shi ${ }^{1}$, \\ Yuchun Jiang ${ }^{1}$, Sijie Zheng ${ }^{1}$, Hui Shao ${ }^{2}$, Naibin Yang ${ }^{3 *}$ and Mingqin Lu ${ }^{1 *}$ \\ 'Department of Infectious Diseases, The First Affiliated Hospital of Wenzhou Medical University, Wenzhou, China, \\ ${ }^{2}$ Department of Infectious Diseases, Taizhou Hospital of Zhejiang Province, Taizhou, China, ${ }^{3}$ Department of Infectious \\ Diseases, Ningbo First Hospital, Ningbo, China
}

Increased protein induced by vitamin $\mathrm{K}$ absence or antagonist-II (PIVKA-II) levels had been widely reported in patients with hepatocellular carcinoma $(\mathrm{HCC})$ and chronic hepatitis. However, the role of PIVKA-II in hepatitis E is unclear. The aim of this study was to clarify the changes related with PIVKA-II and its clinical significance in hepatitis E. We enrolled 84 patients with hepatitis E hospitalized in two hospitals from December 2019 to June 2021. The levels of serum PIVKA-II and related serological indicators in the patients were determined to elucidate the role of PIVKA-II in hepatitis E. We observed that 59.51\% (50/84) of patients showed an increase in PIVKA-II levels. Compared with the normal PIVKA-II group ( $<32 \mathrm{mAU/L}$ ), patients in the elevated PIVKA-II group ( $>32$ $\mathrm{mAU} / \mathrm{L}$ ) had much higher serum total bilirubin (TBIL), direct bilirubin (DBIL), indirect bilirubin (IBIL), and total bile acid (TBA) levels ( $p<0.05$ for each). Compared with the slightly elevated PIVKA-II group (32-125 mAU/L), patients in the significantly elevated PIVKA-II group (>125 mAU/L) had much lower serum albumin, alanine aminotransferase $(A L T)$, aspartate transaminase (AST) levels, and longer days for the hospital stay $(p<0.05$ for each). The association of PIVKA-II with TBIL and DBIL was an inverted U-shaped curve with an inflection point at $199.1 \mathrm{mAU} / \mathrm{L})$. The association of PIVKA-II with IBIL was a U-shaped curve with an inflection point at $18.6 \mathrm{mAU} / \mathrm{L}$ while the association of PIVKAII with albumin was an inverted $U$-shaped curve with an inflection point at $18.6 \mathrm{mAU} / \mathrm{L}$. With the improvement of the disease, PIVKA-II levels were gradually decreased and finally returned to normal. This trend was consistent with that of bilirubin, and a peak appeared in the third week. Therefore, findings from our study show that the increase in PIVKA-II levels can be related to the degree of hepatic insufficiency in patients with hepatitis $E$, wherein PIVKA-II levels are transiently increased, and the trend of change can be related to the disease course.

Keywords: hepatitis E, PIVKA-II, liver function, liver insufficiency, acute hepatitis

\section{INTRODUCTION}

Hepatitis $\mathrm{E}$ is an acute self-limiting disease caused by the hepatitis $\mathrm{E}$ virus (HEV) and is mainly transmitted through the fecal-oral route (1). According to statistics from the WHO, approximately 20 million people worldwide are infected with HEV each year, with more than 3 million cases of acute hepatitis $\mathrm{E}$ and 70,000 patient deaths (2). Thus, hepatitis $\mathrm{E}$ has become one of the most important public health problems worldwide. 
Protein induced by vitamin $\mathrm{K}$ absence or antagonistII (PIVKA-II), or des-gamma-carboxy prothrombin (DCP), is produced when the body is deficient in vitamin $\mathrm{K}$ and prothrombin cannot be shuttled into prothrombin or prothrombin is not fully shuttled, increasing the prothrombin levels and, consequently, PIVKA-II levels (3, 4). PIVKA-II is often used for the auxiliary detection of patients with hepatocellular carcinoma (HCC) accompanied with Alphafetoprotein (AFP) (5). Large scales of studies had analyzed PIVKA-II for patients with HCC using patients with chronic hepatitis as the control group and found slightly elevated PIVKAII in patients with chronic hepatitis (6). We speculated that there might also be an increase of PIVKA-II in acute hepatitis, and the increase might be related to the degree of liver damage. Moreover, the role of PIVKA-II in hepatitis E is still unclear. Thus, we aimed to explore the relationship between PIVKA-II levels and HEV infection in this study, to obtain a better understanding of the role of PIVKA-II in hepatitis E.

\section{MATERIALS AND METHODS}

\section{Patients}

We enrolled 84 patients with hepatitis $\mathrm{E}$ in this study, including 69 patients who were referred to the First Affiliated Hospital of Wenzhou Medical University from December 2019 to June 2021 and 15 patients who were admitted to Taizhou Hospital of Zhejiang Province from December 2019 to June 2021. Inclusion criteria were as followed: HEV infection was diagnosed by testing for anti-HEV immunoglobulin M (IgM) using a serum enzymelinked immunosorbent assay (ELISA) test. Hepatitis E cases were defined based on positive serum anti-HEV IgM, combined with clinical presentation of acute hepatitis (e.g., elevated liver enzymes and/or jaundice and/or non-specific symptoms, such as fatigue, itching, and nausea). The following exclusion criteria were established as previously reported (7): (1) use of antibiotics during the previous month; (2) current bacterial or fungal infections; (3) co-infection with hepatitis A virus, hepatitis B virus, or hepatitis $C$ virus, or the presence of alcoholic fatty liver disease; (4) drug-induced liver disease; (5) autoimmune liver disease; (6) liver cancer, reproductive embryonic cancer, and/or female pregnancy; (7) co-infection with cytomegalovirus or Epstein-Barr virus; (8) presence of metabolic associated fatty liver disease; (9) approval for liver transplantation; and (10) incomplete data.

Studies involving human participants were reviewed and approved by the ethics committee of the First Affiliated Hospital of Wenzhou Medical University and Taizhou Hospital of Zhejiang Province (approval number: no. 2021-zz-162). Due to the retrospective study design involving electronic health records and no additional interventions, written informed consent was waived from the patients or their relatives.

\section{Measurement of the PIVKA-II and Other Serological Indicator Levels}

Serum levels of PIVKA-II were detected using an Abbott I 1000 automatic immunoassay analyzer. Peripheral blood was obtained from each patient, no matter fasting or not. The serum was obtained by centrifuging for $5 \mathrm{~min}$ at $3,000 \mathrm{rpm}$ and stored at $-80^{\circ} \mathrm{C}$ until testing. All serum samples were kept in duplicate. All the operational processes regarding the measurement of PIVKAII were blind to measurers. Detection of HEV IgM via ELISA was performed by Shanghai Kehua Biological Engineering Co., Ltd. Alanine aminotransferase (ALT), aspartate transaminase (AST), alkaline phosphatase (ALP), $\gamma$-glutamyl transpeptidase (GGT), total bilirubin (TBIL), direct bilirubin (DBIL), indirect bilirubin (IBIL), total bile acid (TBA), and albumin levels were analyzed using a Beckman AU5800 automatic biochemical detector. AFP was analyzed using a Beckman DXI800. The prothrombin time (PT) was analyzed using the Stago R Max. The above reagents were all original kits, and the standard operating procedure was strictly followed during the test.

\section{Statistical Analysis}

All statistical analyses were performed using SPSS version 26.0, EmpowerStats (http://www. empowerstats.com), and package R (http://www.Rproject.org). For continuous data, the data were displayed as median (minimum-maximum) or as the actual value of the classification data. Number (\%) was for categorical variables. Baseline features were summarized using descriptive statistics. Groups were compared using chi-square tests for categorical variables, Mann-Whitney $U$ tests were used for continuous variables for comparing two independent groups. A $p<0.05$ was considered to be statistically different.

\section{RESULTS}

\section{Baseline Characteristics Based on Normal or Elevated PIVKA-II}

The flow chart for screening the patients with hepatitis $\mathrm{E}$ is shown in Figure 1. A total of 84 patients with hepatitis E aged 25-77 years were included in our study, with the baseline characteristics according to normal PIVKA-II or elevated PIVKA-II are presented in Table 1 and Figure 2. Of them, 67 were men and 17 were women. The median age was 53 years old, ranging from 25 to 77. Since the range of reference value for PIVKA-II is 11-32 $\mathrm{mAU} / \mathrm{L}$, we adopted $32 \mathrm{mAU} / \mathrm{L}$ as the cut-off value to divide the 84 patients into the normal group $(\mathrm{n}=34)$ with PIVKA-II $\leq 32$ $\mathrm{mAU} / \mathrm{L}$ and elevated group $(\mathrm{n}=50)$ with PIVKA-II $>32 \mathrm{mAU} / \mathrm{L}$. The median level of PIVKA-II in normal group was $24.6 \mathrm{mAU} / \mathrm{L}$ range from 10.6 to $31.4 \mathrm{mAU} / \mathrm{L}$, while their counterpart in the elevated group was much higher as $53.8 \mathrm{mAU} / \mathrm{L}$ with significant statistical differences $(p<0.001)$. Compared with the normal PIVKA-II group, patients in the elevated PIVKA-II group had much higher serum TBIL, DBIL, IBIL, and TBA levels $(p<0.05$ for each). There were no significant differences in the distribution of age and gender between the two groups. Moreover, no significant differences were found in levels of albumin, ALT, AST, ALP, GGT, AFP, PT, and days for the hospital stay.

\section{Association Between Clinical Biochemical Indexes and Degree of Elevated PIVKA-II}

Following Marrero (8), we used $125 \mathrm{mAU} / \mathrm{ml}$ as the grouping cut-off value. Further, we divided the above 50 patients with elevated PIVKA-II levels into two groups according to the degree 
of elevation: the slightly elevated group with PIVKA-II 33-125 $\mathrm{mAU} / \mathrm{ml}$ and the significantly elevated group with PIVKA-II $>125 \mathrm{mAU} / \mathrm{L}$. The median level of PIVKA-II in the significantly elevated group was $318.1 \mathrm{mAU} / \mathrm{ml}$, much higher than their counterpart of $48.8 \mathrm{mAU} / \mathrm{ml}$ in the slightly elevated group $(p$ $<0.001)$. Compared with the slightly elevated PIVKA-II group,

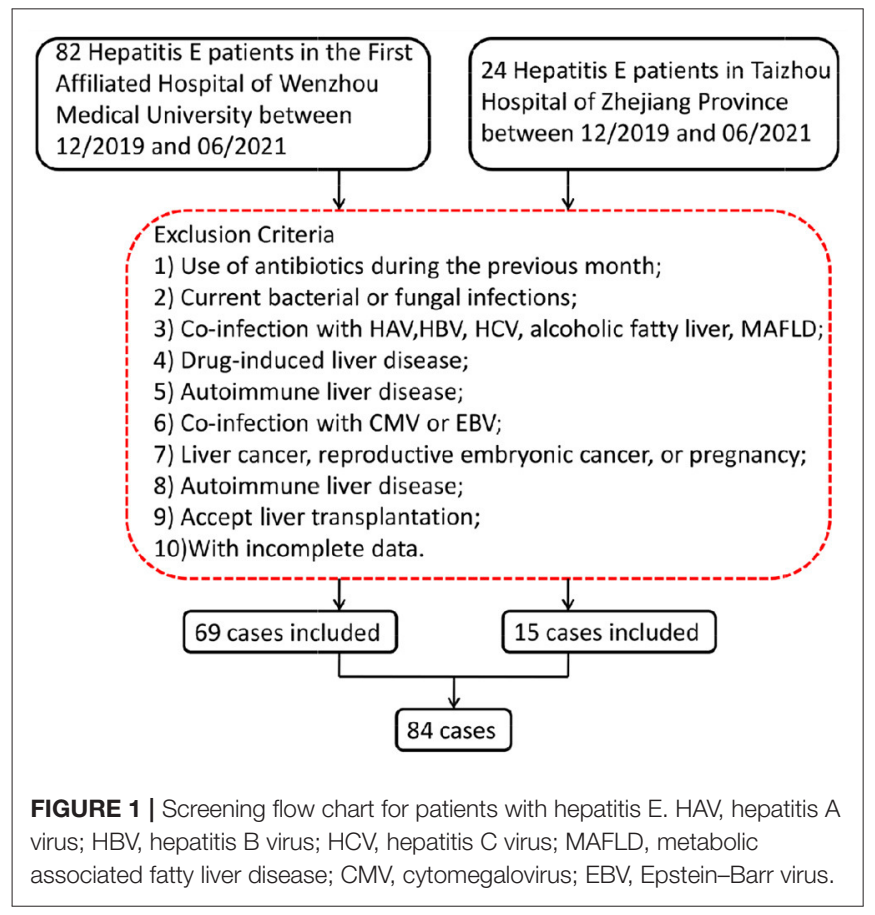

patients in the significantly elevated PIVKA-II group had much lower serum albumin, ALT, AST levels, and longer days for the hospital stay ( $p<0.05$ for each). However, there were no significant differences in the serum TBIL, DBIL, IBIL, ALP, GGT, and TBA levels between the two groups. The detailed information is shown in Table 2 and Figure 2.

\section{Changes of PIVKA-II and Clinical Biochemical Indicators During Hospitalization}

During hospitalization, the serological indicators and PIVKA-II levels of the patients were monitored. As the disease gradually improved, the trend in the PIVKA-II and TB levels was similar; that is, both had peaks that appeared in the third week, which was 2 weeks later than that of transaminase. The peak of AFP appeared was 1 week later than that of PIVKA-II in the fourth week is shown in Table 3.

\section{The Correlation Between PIVKA-II With Bilirubin and Albumin Levels}

The scatter diagram and smooth curve fittings used to characterize the non-linear relationship between PIVKA-II with TBIL, DBIL, IBIL, and albumin levels are shown in Figures 3, 4. PIVKA-II is positively correlated with total bilirubin $(r=0.563$, $p=0.00)$, positively correlated with direct bilirubin $(r=0.556, p$ $=0.00)$, positively correlated with indirect bilirubin $(r=0.357$, $p=0.00)$, and negatively correlated with albumin $(r=-0.264$, $p=0.006)$.

The association between PIVKA-II with TBIL and DBIL was an inverted $\mathrm{U}$-shaped curve, with the point of inflection

TABLE 1 | Baseline characteristics of patients with hepatitis E.

\begin{tabular}{|c|c|c|c|c|}
\hline Parameter & Total $(n=84)$ & Normal group $(n=34)$ & Elevated group $(n=50)$ & $P$ value \\
\hline Age (years) & $53(25-77)$ & $52(29-77)$ & $52(25-74)$ & 0.616 \\
\hline Gender & & & & 0.084 \\
\hline Male & 67 (79.8\%) & 24 (70.6\%) & 43 (86.0\%) & \\
\hline Female & 17 (20.2\%) & $10(29.4 \%)$ & 7 (14.0\%) & \\
\hline TBIL ( $\mu \mathrm{mol} / \mathrm{L})$ & $115.5(7-460.3)$ & $42.0(7.0-236.0)$ & 120.5 (12.0-460.3) & $<0.001$ \\
\hline DBIL ( $\mu \mathrm{mol} / \mathrm{L})$ & $94.6(2-396)$ & $27.8(2.0-232.0)$ & 95.5 (6.0-396.0) & $<0.001$ \\
\hline IBIL ( $\mu \mathrm{mol} / \mathrm{L})$ & $21.0(4-164.7)$ & $10.6(4.0-77.9)$ & $16.0(4.0-164.7)$ & 0.015 \\
\hline Albumin (g/L) & $35.8(23.9-46.4)$ & 36.2 (23.9-46.4) & 35.5 (27.0-45.6) & 0.512 \\
\hline ALT (U/L) & $1,405.14(25-5,406)$ & $724.0(25-5,406)$ & 1,217.0 (32.0-4,228.0) & 0.219 \\
\hline AST (U/L) & $772.7(22-6,436)$ & $166.0(22-6,436)$ & $382.5(33.0-5,720.0)$ & 0.111 \\
\hline ALP (U/L) & $208.8(61-475)$ & $174.0(61.0-404.0)$ & $201.0(84.0-475.0)$ & 0.155 \\
\hline GGT (U/L) & 276.4 (31-903) & 232.5 (34.0-640.0) & 209.5 (31.0-903.0) & 0.781 \\
\hline TBA ( $\mu \mathrm{mol} / \mathrm{L})$ & $168.1(2-477)$ & $104.1(2.0-477.0)$ & 238.5 (2.4-450.0) & 0.039 \\
\hline AFP (ng/mL) & $46.4(1.22-474.0)$ & $33.2(1.2-340.1)$ & $20.4(1.5-474.0)$ & 0.830 \\
\hline PT (s) & $21.5(10-337)$ & $14.0(12.2-337.0)$ & $13.5(10.0-19.8)$ & 0.247 \\
\hline PIVIK-II (mAU/L) & $66.7(10.6-546.1)$ & $24.6(10.6-31.4)$ & $53.8(33.0-9,235.0)$ & $<0.001$ \\
\hline Hospital days & $13.7(4-36)$ & $10.5(4.0-33.0)$ & $13.0(7.0-36.0)$ & 0.085 \\
\hline
\end{tabular}

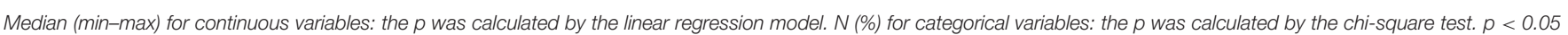

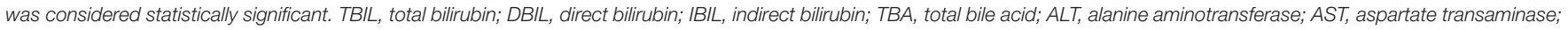
GGT, $\gamma$-glutamyl transpeptidase; TBA, total bile acid; AFP, Alpha-fetoprotein. 
identified using a two piecewise linear regression model, at $199.1 \mathrm{mAU} / \mathrm{ml}$ (Table 4). For a PIVKA-II < $199.1 \mathrm{mAU} / \mathrm{ml}$, every $1 \mathrm{mAU} / \mathrm{ml}$ increase in PIVKA-II was associated with a $1.6 \mu \mathrm{mol} / \mathrm{L}$ greater TBIL (95\% CI: $1.1-2.0$ ); by comparison, for individuals with a PIVKA-II $>199.1 \mathrm{mAU} / \mathrm{ml}$, a $1 \mathrm{mAU} / \mathrm{ml}$ increase in PIVKA-II was associated with a $0.7 \mu \mathrm{mol} / \mathrm{L}$ decrease in TBIL (95\% CI: -1.1 to -0.2 ). Similarly, for a PIVKA-II $<199.1 \mathrm{mAU} / \mathrm{ml}$, every $1 \mathrm{mAU} / \mathrm{ml}$ increase in PIVKA-II was associated with a $1.4 \mu \mathrm{mol} / \mathrm{L}$ greater DBIL (95\% CI: $1.0-1.8$ ); by comparison, for individuals with a PIVKA-II $>199.1 \mathrm{mAU} / \mathrm{ml}$, a $1 \mathrm{mAU} / \mathrm{ml}$ increase in PIVKA-II was associated with a 0.6 $\mu \mathrm{mol} / \mathrm{L}$ decrease in DBIL (95\% CI: -1.0 to -0.3 ).

The association between PIVKA-II with IBIL was a U-shaped curve, with the point of inflection identified using a two piecewise

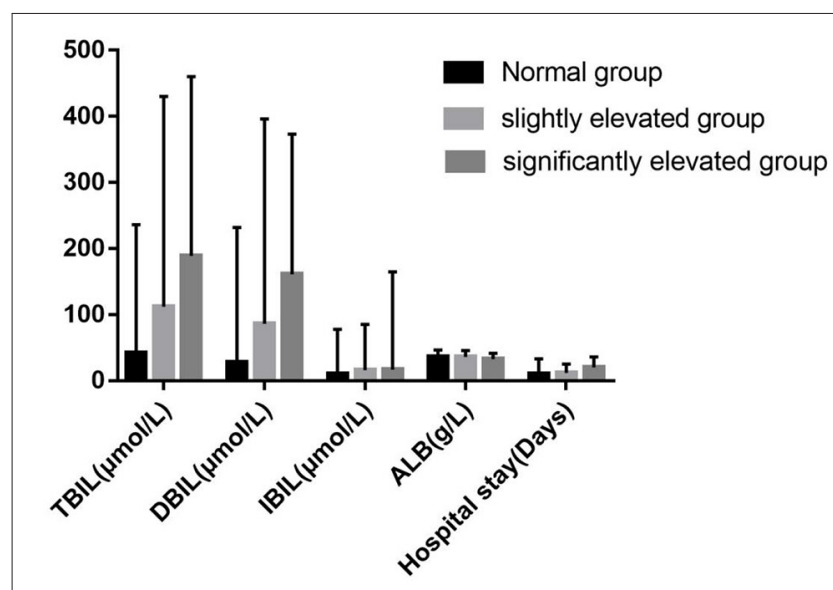

FIGURE 2 | Levels of clinical biochemical indexes in different groups of serum PIVKA-II levels. PIVKA, protein induced by vitamin $\mathrm{K}$ absence or antagonist-II. linear regression model, at $18.6 \mathrm{mAU} / \mathrm{ml}$ (Table 4). For a PIVKAII $<18.6 \mathrm{mAU} / \mathrm{ml}$, every $1 \mathrm{mAU} / \mathrm{ml}$ increase in PIVKA-II was associated with a $5.4 \mu \mathrm{mol} / \mathrm{L}$ lower IBIL (95\% CI: -9.8 to -0.9 ); by comparison, for individuals with a PIVKA-II $>18.6$ $\mathrm{mAU} / \mathrm{ml}$, a $1 \mathrm{mAU} / \mathrm{ml}$ increase in PIVKA-II was associated with a $0.1 \mu \mathrm{mol} / \mathrm{L}$ increase in IBIL (95\% CI: 0.0-0.1). However, the association between PIVKA-II with albumin was an inverted Ushaped curve, with the point of inflection identified using a two piecewise linear regression model, at $18.6 \mathrm{mAU} / \mathrm{ml}$ (Table 4). For a PIVKA-II $<18.6 \mathrm{mAU} / \mathrm{ml}$, every $1 \mathrm{mAU} / \mathrm{ml}$ increase in PIVKA-II was associated with a $1.1 \mu \mathrm{mol} / \mathrm{L}$ greater albumin $(95 \%$ CI: 0.2-2.0).

\section{DISCUSSION}

Hepatitis $\mathrm{E}$ is distributed worldwide and is prevalent in many developing countries in Africa and Asia (9). In fact, the WHO has considered hepatitis $\mathrm{E}$ as an important public health problem in developing countries (2). In the recent years, with the improvement of the living standards of the Chinese population, the hepatitis E epidemic has been more controlled than that before, but there are still sporadic cases, and small-scale hepatitis E outbreaks have been reported from time to time (10-12). In addition, the age of onset has become a new feature in the epidemiology of hepatitis $\mathrm{E}$ in the country. An epidemiological survey on hepatitis $\mathrm{E}$ in China showed that the number of reported cases aged 45-69 years accounted for more than half of all reported cases (10). Moreover, Li et al. reported that the prevalence of anti-HEV antibodies had increased with age (13). Most cases of hepatitis E are self-limiting, and progression to acute liver failure is rare. However, older and diabetic patients are more likely to develop acute liver failure and die when compared with younger and non-diabetic patients (14). Pregnant women, especially those in their last trimester, had a case fatality

TABLE 2 | Association between clinical biochemical indexes and degree of elevated PIVKA-II.

\begin{tabular}{|c|c|c|c|}
\hline Parameter & $\begin{array}{l}\text { slightly elevated } \\
\text { (<125 mAU/L) }\end{array}$ & $\begin{array}{c}\text { significantly elevated } \\
\text { (>125 } \mathrm{mAU} / \mathrm{L})\end{array}$ & $P$ value \\
\hline PIVKA-II (mAU/L) & $48.8(33.0-124.6)$ & $318.1(127.2-9,235.0)$ & $<0.001$ \\
\hline TBIL ( $\mu \mathrm{mol} / \mathrm{L})$ & $112.0(12.0-430.0)$ & $189.0(45.0-460.3)$ & 0.050 \\
\hline DBIL ( $\mu \mathrm{mol} / \mathrm{L})$ & $86.0(6.0-396.0)$ & $161.0(37.0-373.0)$ & 0.037 \\
\hline IBIL ( $\mu \mathrm{mol} / \mathrm{L})$ & $16.0(4.0-85.3)$ & $17.0(8.0-164.7)$ & 0.390 \\
\hline Albumin (g/L) & $36.2(27.6-45.6)$ & $32.5(27.0-41.3)$ & 0.018 \\
\hline ALT (U/L) & 1,461.0 (97.0-4,228.0) & $237.0(32.0-3,203.0)$ & 0.002 \\
\hline AST (U/L) & $564.0(44.0-5,720.0)$ & 89.0 (33.0-1,294.0) & 0.008 \\
\hline ALP (U/L) & 200.0 (84.0-466.0) & 208.5 (115.0-475.0) & 0.921 \\
\hline GGT (U/L) & 219.0 (36.0-840.0) & 150.0 (31.0-903.0) & 0.181 \\
\hline TBA ( $\mu \mathrm{mol} / \mathrm{L})$ & $241.0(2.4-450.0)$ & 202.7 (17.0-264.0) & 0.431 \\
\hline AFP (ng/mL) & $15.9(1.5-474.0)$ & $28.4(4.2-455.2)$ & 0.383 \\
\hline PT (s) & $13.4(1.0-18.0)$ & $15.0(12.3-19.8)$ & 0.165 \\
\hline Hospital stay (Days) & $12.0(7.0-25.0)$ & $20.0(10.0-36.0)$ & 0.005 \\
\hline
\end{tabular}

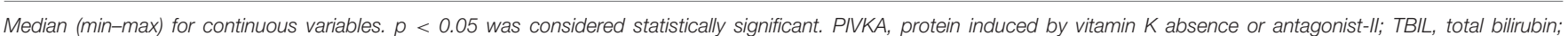

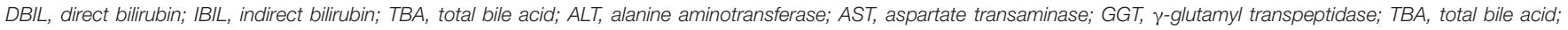
AFP, Alpha-fetoprotein. 
TABLE 3 | Changes in PIVKA-II and clinical biochemical indicators during hospitalization.

\begin{tabular}{|c|c|c|c|c|c|}
\hline Time (weeks) & $<1$ & $1-2$ & $2-3$ & $3-4$ & $>4$ \\
\hline PIVKA-II (mAu/mL) & $45.8(38.7-369.8)$ & 74.7 (31.7-534.7) & 1,194.3 (616.6-2,622.1) & $41.1(20.0-885.3)$ & $36.2(29.2-116.0)$ \\
\hline TBIL $(\mu \mathrm{mol} / \mathrm{L})$ & $135.3(7.0-460.3)$ & $160.2(16.1-242)$ & $245.3(160-357)$ & $245.0(41.7-425)$ & $37.8(21-50.7)$ \\
\hline DBIL ( $\mu \mathrm{mol} / \mathrm{L})$ & $107.8(2.0-396.0)$ & $130.2(7-223)$ & $216.5(141-318)$ & $212.3(23.7-363)$ & $21.8(10.7-31.1)$ \\
\hline IBIL $(\mu \mathrm{mol} / \mathrm{L})$ & $27.5(2-164.7)$ & $12.4(4-19)$ & $28.8(19-81)$ & $32.8(11-62)$ & $15.9(10.3-19.6)$ \\
\hline ALP (U/L) & $232.8(61-475)$ & $104.5(95-123)$ & $141.3(120-166)$ & $117.0(109-143)$ & $123.0(95-131)$ \\
\hline GGT (U/L) & 150.0 (91.3-443.3) & 104.0 (72.0-160.5) & $66.5(51.7-88.5)$ & $71.0(66.5-143.5)$ & $31.5(21.0-45.5)$ \\
\hline TBA $(\mu \mathrm{mol} / \mathrm{L})$ & $210.4(2-477)$ & $142.6(12.1-273)$ & $239.7(177.4-363)$ & $41.7(41.7-41.7)$ & $8.8(4.5-13.2)$ \\
\hline AFP (ng/ml) & $7.1(1.8-27.8)$ & $6.5(4.1-1,032.1)$ & $132.6(41.8-396.4)$ & $148.6(8.1-119.1)$ & $88.7(7.8-98.0)$ \\
\hline PT (s) & $15.7(1.0-337)$ & $14.1(13.5-14.9)$ & $13.8(13.4-14.3)$ & $17.1(13.1-20.7)$ & $13.1(13.1-13.1)$ \\
\hline Albumin (g/L) & 34.4 (31.9-38.5) & $33.3(28.9-36.3)$ & $30.8(30.3-34.0)$ & $36.0(34.3-41.1)$ & $36.8(36.5-38.4)$ \\
\hline
\end{tabular}

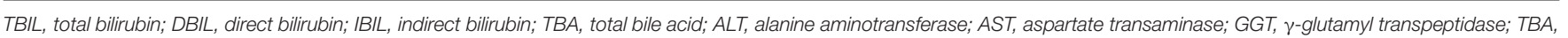
total bile acid; AFP, Alpha-fetoprotein.

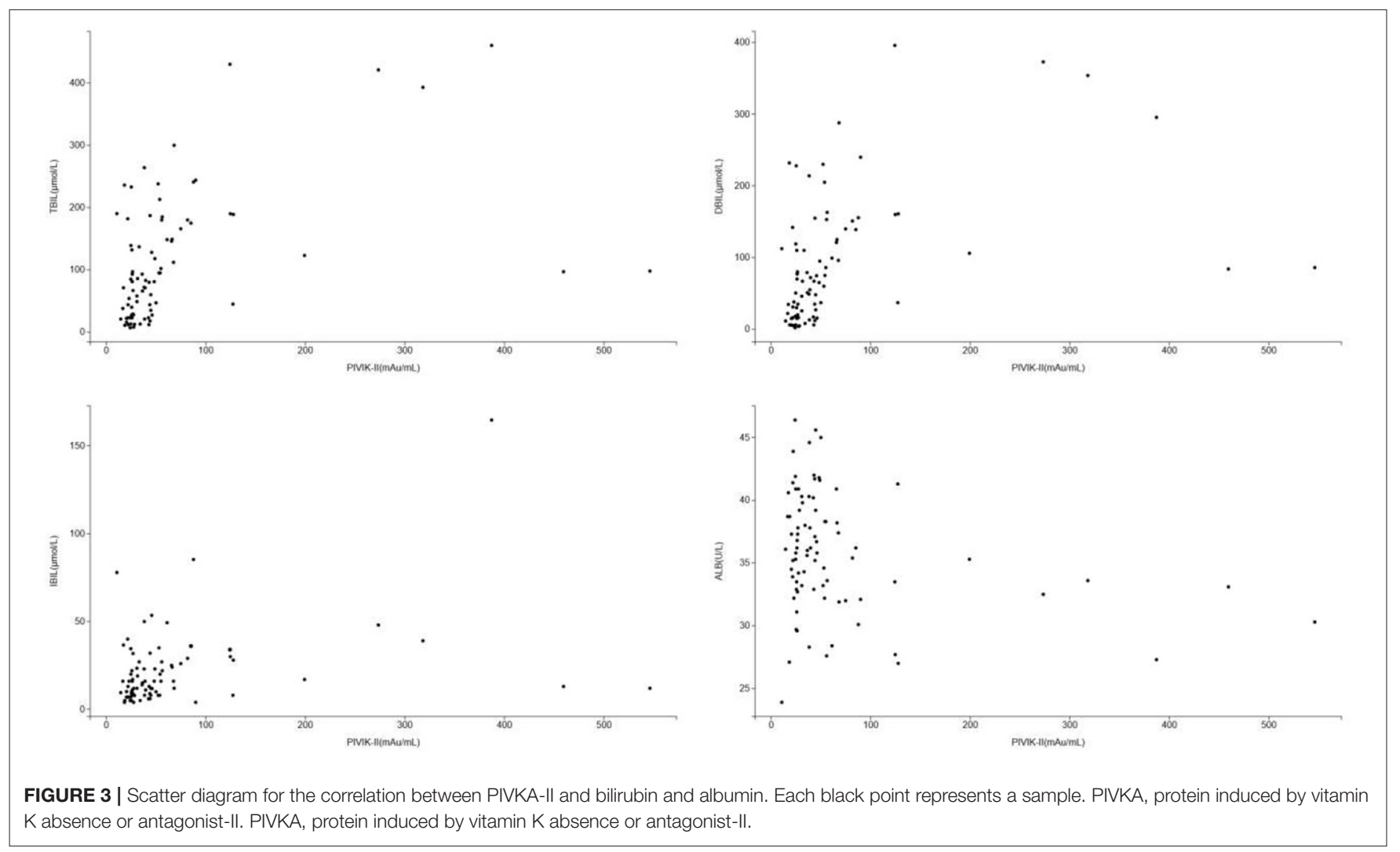

rate of more than $10 \%(15,16)$. In our current study, these patients had a median value of 53 years old ranged from 25 to 77 . There were no significant differences in age distribution between the normal PIVKA-II group and the elevated PIVKAII group. Pregnant patients were not included in our study and none of the 84 patients progressed to acute liver failure during hospitalization.
Protein induced by vitamin $\mathrm{K}$ absence or antagonist-II -II is a protein produced due to vitamin $\mathrm{K}$ deficiency or antagonists and is mainly composed of liver cell particles. Carboxylase system and epoxide reductase work together to produce PIVKA-II. The glutamic acid residues in the $\gamma$-carboxyglutamate structure are not fully carboxylated to $\gamma$-carboxyglutamate and lose their normal activity $(17,18)$. PIVKA-II has been recently found to 

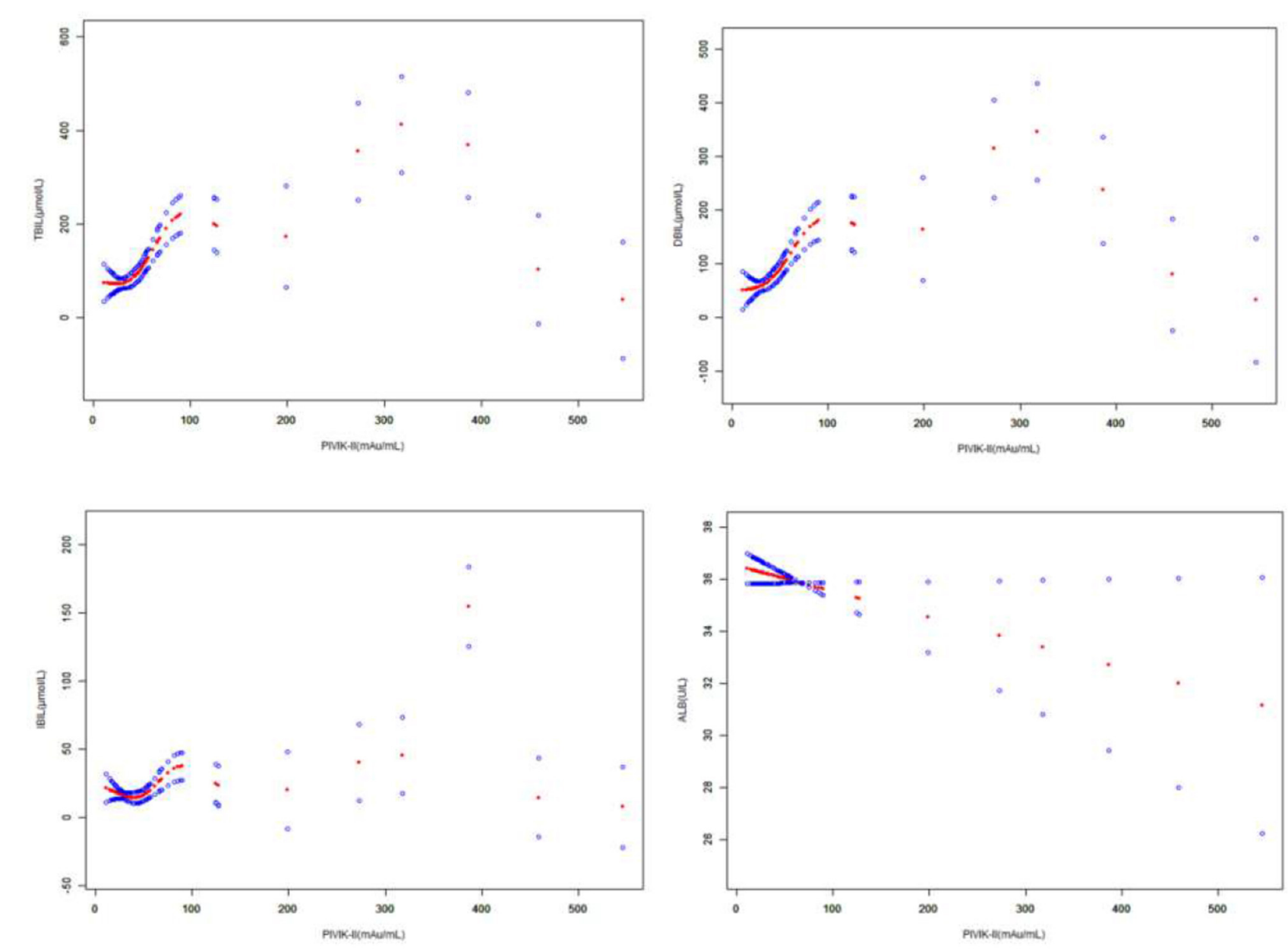

FIGURE 4 | The correlation between PIVKA-II and bilirubin and albumin. The solid red line represents the smooth curve fit between variables. Blue bands represent the $95 \% \mathrm{Cl}$ from the fit. Gender and age were adjusted. PIVKA, protein induced by vitamin K absence or antagonist-II.

have a good diagnostic value for early liver tumors, especially HCC (19). However, PIVKA-II elevation is not specific for HCC diagnosis, reported in many other disease situations. Moreover, $\mathrm{Wu}$ et al. reported that PIVKA-II levels in noncirrhotic chronic hepatitis B patients were higher than that in the healthy control group (20). Other factors, such as vitamin $\mathrm{K}$ deficiency, taking warfarin, primary gastric adenocarcinoma, transplant rejection, lack of nutrition, intestinal flora imbalance, renal failure, inflammatory bowel disease, and alcoholic liver disease, led to increased serum PIVKA-II levels in non-HCC patients (6). Besides, Takikawa reported that PIVKA-II helps to monitor the severity of acute liver injury (21). In this study, we included 84 cases of patients with hepatitis $\mathrm{E}$ and measured the levels of the PIVKA-II and various serological indicators, and monitored the indicators during hospitalization. The PIVKA-II levels increased in $59.51 \%(50 / 84)$ of patients with hepatitis E, four cases had PIVKA-II levels $>1,000 \mathrm{mAU} / \mathrm{ml}$, the highest of which was $9,235 \mathrm{mAU} / \mathrm{ml}$. We classified the degree of increase in the PIVKA-II levels into two groups. Compared with the slightly elevated PIVKA-II group, patients in the significantly elevated PIVKA-II group had much lower serum albumin, ALT, AST levels, and longer days for the hospital stay. An elevated PIVKA-II correlated with a greater TBIL, DBIL, IBIL, and lower albumin. Moreover, we identified a non-linear relationship between PIVKA-II with TBIL and DBIL with a point of inflection at $199.1 \mathrm{mAU} / \mathrm{ml}$ and a non-linear relationship between PIVKAII with IBIL and albumin with a point of inflection at 18.6 $\mathrm{mAU} / \mathrm{ml}$. These results indicated that PIVKA-II levels might be related with the severity of hepatitis $\mathrm{E}$.

An increase in prothrombin levels also leads to the production of abnormal prothrombin (18). Therefore, we believe that PIVKA-II may be associated with the severity of acute liver damage. Actually, the underlying mechanism behind the effect of HEV infection on serum PIVKA-II levels remains unclear. We believe that this may be related to liver cell damage and metabolic disorders in patients with hepatitis $\mathrm{E}$, which decreased the ability of liver cells to synthesize protein and may weaken the function of vitamin K-dependent shuttling 
TABLE 4 | Threshold effect analysis of PIVKA-II on TBIL, DBIL, IBIL, and albumin using the two-piecewise linear regression model.

\begin{tabular}{|c|c|}
\hline Parameters & Adjusted $\beta(95 \% \mathrm{Cl}), P$-value \\
\hline \multicolumn{2}{|l|}{ TBIL } \\
\hline Fitting by the standard linear model & $0.4(0.2,0.6),<0.001$ \\
\hline \multicolumn{2}{|l|}{ Fitting by the two-piecewise linear model } \\
\hline Inflection point & 199.1 \\
\hline PIVKA-II $<199.1(\mathrm{mAU} / \mathrm{mL})$ & $1.6(1.1,2.0),<0.001$ \\
\hline PIVKA-II >199.1 (mAU/mL) & $-0.7(-1.1,-0.2), 0.003$ \\
\hline Log likelihood ratio & $<0.001$ \\
\hline \multicolumn{2}{|l|}{ DBIL } \\
\hline Fitting by the standard linear model & $0.3(0.1,0.5) 0.002$ \\
\hline \multicolumn{2}{|l|}{ Fitting by the two-piecewise linear model } \\
\hline Inflection point & 199.1 \\
\hline PIVKA-\|l $<199.1(\mathrm{mAU} / \mathrm{mL})$ & $1.4(1.0,1.8)<0.001$ \\
\hline PIVKA-II >199.1 (mAU/mL) & $-0.6(-1.0,-0.3) 0.001$ \\
\hline Log likelihood ratio & $<0.001$ \\
\hline \multicolumn{2}{|l|}{ IBIL } \\
\hline Fitting by the standard linear model & $(0.0,0.1) 0.006$ \\
\hline \multicolumn{2}{|l|}{ Fitting by the two-piecewise linear model } \\
\hline Inflection point & 18.6 \\
\hline $\begin{array}{l}\text { PIVKA-II< } 18.6(\mathrm{mAU} / \mathrm{mL}) \\
\text { PIVKA-II>18.6 (mAU/mL) } \\
\text { Log likelihood ratio }\end{array}$ & $\begin{array}{l}-5.4(-9.8,-0.9) 0.020 \\
0.1(0.0,0.1) 0.0020 .014\end{array}$ \\
\hline \multicolumn{2}{|l|}{ Albumin } \\
\hline \multicolumn{2}{|l|}{ Fitting by the standard linear model } \\
\hline Fitting by the two-piecewise linear mode & $-0.0(-0.0,0.0) 0.064$ \\
\hline Inflection point & 18.6 \\
\hline PIVKA-II < 18.6 (mAU/mL) & $1.1(0.2,2.0) 0.017$ \\
\hline $\begin{array}{l}\text { PIVKA-II > 18.6 (mAU/mL) } \\
\text { Log likelihood ratio }\end{array}$ & $-0.0(-0.0,-0.0) 0.0260 .013$ \\
\hline
\end{tabular}

Gender and age were adjusted. PIVKA, protein induced by vitamin $K$ absence or antagonist-II.

enzymes, causing the metabolic utilization of vitamin $\mathrm{K}$. Besides, elevated PIVKA-II levels in hepatitis E may also be related with Vitamin $\mathrm{K}$ absence. Regretfully, the relationship between serum vitamin $\mathrm{K}$ concentration and serum PIVKAII levels was not explored in our current study because data on serum vitamin $\mathrm{K}$ levels are unavailable. Moreover, no related pieces of literature were previously published about the relationship between Vitamin $\mathrm{K}$ absence and $\mathrm{HEV}$ infection. Therefore, whether Vitamin $\mathrm{K}$ absence existed in patients with $\mathrm{HEV}$ infection was still unclear and the effect of $\mathrm{HEV}$ infection on serum PIVKA-II levels needs to be clarified in future research.

With the gradual recovery of patients with hepatitis E, PIVKAII levels also gradually decreased, and the peak was delayed by 2 weeks compared with the peak of transaminase, which was roughly similar to that of bilirubin. The peak of AFP was 1 week later than that of PIVKA-II. Many studies believe that AFP exists in the cytoplasm of oval cells or hyperproliferative cells $(22,23)$, which indicates the proliferation of liver cells after injury, and the new liver cells may synthesize AFP briefly in the early stage. We believe that PIVKA-II is related to liver cell damage, so there may be a certain node in the abnormal metabolism and hyperplasia of liver cell necrosis, which is a turning point in the disease of the patients with hepatitis E. However, we did not have enough cases in this study, and the sample size needs to be further expanded.

Based on the above information, it could be seen that more than half of patients with hepatitis E had elevated PIVKAII levels, which were all transiently elevated, and the patients gradually recovered after a few weeks. With the recovery of patients from hepatitis $\mathrm{E}$, none of the cases continued to progress. Therefore, PIVKA-II could be a significant reference for the course of hepatitis E disease. Moreover, we can refer to the PIVKA-II level of patients with hepatitis $\mathrm{E}$ to assess the severity of the patient's condition and the trend of the outcome to assist in the diagnosis and treatment of the disease further. If the patient's PIVKA-II continues to rise, further examinations should be undertaken, and the possibility of HCC should be ruled out. The association between PIVKA-II with TBIL, DBIL, IBIL, and albumin was a U-shaped or inverted U-shaped curve with an obvious point of inflection. However, this study had some limitations. First, this was a retrospective study. The data collection time was relatively short. The study population was recruited from only two centers. We need to conduct more cases and perform long-term follow-up evaluations. Second, we excluded individuals with HCC from our study sample as HCC may have a significant influence on PIVKA-II. Third, there remains the possibility of bias caused by other potential confounding factors that we did not adjust for.

\section{DATA AVAILABILITY STATEMENT}

The research involves patient private information, the data that support the findings of this study are available from the corresponding author upon reasonable request.

\section{ETHICS STATEMENT}

The studies involving human participants were reviewed and approved by the Ethics Committee of the First Affiliated Hospital of Wenzhou Medical University and Taizhou Hospital of Zhejiang Province (approval number: No. 2021-zz-162). Due to the retrospective study design involving electronic health records and no additional interventions, written informed consent was waived from the patients or their relatives.

\section{AUTHOR CONTRIBUTIONS}

YC, YY, NY, and MLu contributed to the conception and design of the study. YC organized the database. YY performed the statistical analysis. YC wrote the first draft of the manuscript. SL, MLi, XX, HS, YJ, SZ, and HS wrote sections of the manuscript. All the authors contributed to manuscript revision, read, and approved the submitted version. 


\section{REFERENCES}

1. Wang Y, Zhou X, Debing Y, Chen K, Van Der Laan LJ, Neyts J, et al. Calcineurin inhibitors stimulate and mycophenolic acid inhibits replication of hepatitis E virus. Gastroenterology. (2014) 146:1775-83. doi: 10.1053/j.gastro.2014.02.036

2. Nimgaonkar I, Ding Q, Schwartz RE, Ploss A. Hepatitis E virus: advances and challenges. Nat Rev Gastroenterol Hepatol. (2018) 15:96-110. doi: $10.1038 /$ nrgastro.2017.150

3. Omata M, Cheng AL, Kokudo N, Kudo M, Lee JM, Jia J, et al. Asia-Pacific clinical practice guidelines on the management of hepatocellular carcinoma: a 2017 update. Hepatol Int. (2017) 11:317-70. doi: 10.1007/s12072-017-9799-9

4. Svobodova S, Karlikova M, Topolcan O, Pecen L, Pestova M, Kott O, et al. PIVKA-II as a potential new biomarker for hepatocellular carcinoma - a pilot study. In vivo (Athens, Greece). (2018) 32:1551-54. doi: 10.21873/invivo.11413

5. Marrero JA, Feng Z, Wang Y, Nguyen MH, Befeler AS, Roberts LR, et al. Alpha-fetoprotein, des-gamma carboxyprothrombin, and lectin-bound alpha-fetoprotein in early hepatocellular carcinoma. Gastroenterology. (2009) 137:110-8. doi: 10.1053/j.gastro.2009.04.005

6. Kang KH, Kim JH, Kang SH, Lee BJ, Seo YS, Yim HJ, et al. The influence of alcoholic liver disease on serum PIVKA-II levels in patients without hepatocellular carcinoma. Gut and liver. (2015) 9:224-30. doi: $10.5009 /$ gnl14047

7. Wu J, Huang F, Ling ZX, Liu SC, Liu J, Fan J, et al. Altered faecal microbiota on the expression of Th cells responses in the exacerbation of patients with hepatitis E infection. J Viral Hepat. (2020) 27:1243-52. doi: 10.1111/jvh.13344

8. Marrero JA, Su GL, Wei W, Emick D, Conjeevaram HS, Fontana RJ, et al. Desgamma carboxyprothrombin can differentiate hepatocellular carcinoma from nonmalignant chronic liver disease in american patients. Hepatology. (2003) 37:1114-21. doi: 10.1053/jhep.2003.50195

9. Kirkwood CD, Dobscha KR, Steele AD. Hepatitis E should be a global public health priority: recommendations for improving surveillance and prevention. Expert Rev Vaccines. (2020) 19:1129-40. doi: 10.1080/14760584.2020.1874930

10. Yu SS, Rui J, Cheng XQ, Zhao ZY, Liu C, Lin SN, et al. Hepatitis $\mathrm{E}$ in 24 Chinese cities, 2008-2018: a new analysis method for the disease's occupational characteristics. Front Public Health. (2021) 9:720953. doi: 10.3389/fpubh.2021.720953

11. Su Q, Liu YQ, Cui ZZ, Chang S, Zhao P. Genetic diversity of avian hepatitis E virus in China, 2018-2019. Transbound Emerg Dis. (2020) 67:2403-07. doi: $10.1111 /$ tbed.13578

12. Lu J, Li Q, Jiang JY, Li ZQ, Wang PY, Sheng ZK, et al. Laboratorybased surveillance and clinical profile of sporadic hev infection in Shanghai, China. Virol Sin. (2021) 36:644-54. 12250-020-00336w doi: 10.1007/s12250-020-00336-w

13. Li RC, Ge SX, Li YP, Zheng YJ, Nong Y, Guo QS, et al. Seroprevalence of hepatitis E virus infection, rural southern People's Republic of China. Emerging infectious diseases. (2006) 12:1682-8. doi: 10.3201/eid1211. 060332

14. Wu J, Guo NZ, Zhang XY, Xiong CQ, Liu J, Xu YP, et al. HEV-LF (S) : A novel scoring model for patients with hepatitis $\mathrm{E}$ virus-related liver failure. J Viral Hepat. (2019) 26:1334-43. doi: 10.1111/jvh. 13174

15. Terrault NA, Levy MT, Cheung KW, Jourdain G. Viral hepatitis and pregnancy. Nat Rev Gastroenterol Hepatol. (2021) 18:117-30. doi: 10.1038/s41575-020-00361-w

16. Zhang F, Wang JF, Cheng JL, Zhang XH, He QY, Liang ZZ, et al. Clinical features of sporadic hepatitis $\mathrm{E}$ virus infection in pregnant women in Shanghai, China. J Infect. (2021) 21:4. doi: 10.1016/j.jinf.2021.11.004

17. Zhang YS, Chu JH, Cui SX, Song ZY, Qu XJ. Des- $\gamma$-carboxy prothrombin (DCP) as a potential autologous growth factor for the development of hepatocellular carcinoma. Cell Physiol Biochemistry. (2014) 34:903-15. doi: $10.1159 / 000366308$

18. Inagaki Y, Tang W, Makuuchi M, Hasegawa K, Sugawara Y, Kokudo N. Clinical and molecular insights into the hepatocellular carcinoma tumour marker des- $\gamma$-carboxyprothrombin. Liver Int. (2011) 31:22-35. doi: 10.1111/j.1478-3231.2010.02348.x

19. Clinical Practice Guidelines for Hepatocellular Carcinoma Differ between Japan, United States, and Europe. Liver cancer. (2015) 4:85-95. doi: 10.1159/000367730

20. Wu JL, Xiang ZY, Bai L, He LG, Tan L, Hu M, et al. Diagnostic value of serum PIVKA-II levels for BCLC early hepatocellular carcinoma and correlation with HBV DNA. Cancer Biomark. (2018) 23:235-42. doi: 10.3233/CBM-181402

21. Takikawa Y. Abnormal prothrombin in acute hepatic failure: the characterization and clinical evaluation. Nihon Shokakibyo Gakkai Zasshi. (1991) 88:1074-82

22. Xu Y, Guo QL, Wei LB. The emerging influences of alpha-fetoprotein in the tumorigenesis and progression of hepatocellular carcinoma. Cancers. (2021) 13:5096 doi: 10.3390/cancers13205096

23. Daniele B, Bencivenga A, Megna AS, Tinessa V. Alpha-fetoprotein and ultrasonography screening for hepatocellular carcinoma. Gastroenterology. (2004) 127:S108-12. doi: 10.1053/j.gastro.2004.09.023

Conflict of Interest: The authors declare that the research was conducted in the absence of any commercial or financial relationships that could be construed as a potential conflict of interest.

Publisher's Note: All claims expressed in this article are solely those of the authors and do not necessarily represent those of their affiliated organizations, or those of the publisher, the editors and the reviewers. Any product that may be evaluated in this article, or claim that may be made by its manufacturer, is not guaranteed or endorsed by the publisher.

Copyright (c) 2022 Chen, Yang, Li, Lin, Xie, Shi, Jiang, Zheng, Shao, Yang and Lu. This is an open-access article distributed under the terms of the Creative Commons Attribution License (CC BY). The use, distribution or reproduction in other forums is permitted, provided the original author(s) and the copyright owner(s) are credited and that the original publication in this journal is cited, in accordance with accepted academic practice. No use, distribution or reproduction is permitted which does not comply with these terms. 